\section{Molecular basis and clinical management of Pompe disease}

\author{
Giancarlo Parenti, ${ }^{1,2}$ Giuseppe Di lorio, ${ }^{3}$ \\ Simone Sampaolo, ${ }^{3}$ \\ Giuseppe Fiorentino, ${ }^{4}$ Vincenzo Farina, ${ }^{5}$ \\ Simona Fecarotta, ${ }^{1}$ Fabio Valente, ${ }^{6}$ \\ Serena Ascione, ${ }^{1}$ Mario Caputi, ${ }^{4}$ \\ Generoso Andria ${ }^{1}$ \\ 1 Department of Translational Medical \\ Sciences, Federico II University; ${ }^{2}$ Telethon \\ Institute of Genetics and Medicine; \\ ${ }^{3}$ Department of Neurology, Second \\ University of Naples; ${ }^{4}$ Respiratory \\ Disease Pathophysiology and \\ Rehabilitation Unit, Monaldi Hospital, \\ AO Colli, Second University of Naples; \\ ${ }^{5}$ Department of Pediatrics, Paediatric \\ Cardiology, Federico II University; \\ ${ }^{6}$ Cardiology Unit, Monaldi Hospital, AO \\ Colli, Second University of Naples, Italy
}

\section{Abstract}

Pompe disease (glycogenosis type II) is a rare autosomal recessive lysosomal storage disorder due to mutations of the $G A A$ gene, leading to the deficiency of acid $\alpha$-glucosidase and consequent glycogen storage in various tissues, mainly in the skeletal muscle, heart and liver. The consequent clinical picture is mainly due to the muscle and heart involvement, although clinical manifestations may be multi-systemic.

The phenotype of patients is heterogeneous and the severity is inversely related to the residual enzymatic activity of acid $\alpha$-glucosidase. More than 200 different mutations of $G A A$ gene have been described and genotype/phenotype correlations have been established for some of them.

Traditionally three forms have been described, i.e. early onset classical and non-classical forms and late onset attenuated forms.

A severe hypertrophic cardiomyopathy in combination with conduction disorder in newborns represents a typical feature in the classic infantile presentation, while clinical picture in late onset forms is dominated by skeletal muscle dysfunction, resulting in mobility and respiratory problems.

Enzyme replacement therapy with recombinant human GAA is the approved therapeutic approach in Pompe disease patients. Clinical trials on enzyme replacement therapy (ERT) support the efficacy in improving survival and hypertrophic cardiomyopathy, while efficacy seems to be variable on manifestations due to skeletal muscle involvement, mainly in late- onset patients. Considering the limitations of ERT and its high costs, innovative therapeutic approaches are now under development.

\section{Introduction}

Pompe disease (PD, glycogen storage disease type II) is a rare metabolic myopathy, with an autosomal recessive mode of inheritance, caused by mutations of the acid $\alpha$-glucosidase $(G A A)$ gene, resulting in the deficiency of the lysosomal hydrolase acid $\alpha$-glucosidase (GAA, acid maltase, E.C.3.2.1.20). PD is characterized by generalized storage of glycogen, particularly in the heart, skeletal muscles and liver. An overall incidence of 1 in 40,000 live birth, with different ethnical frequencies has been reported.

The disease is characterized by broad phenotypic heterogeneity and varying levels of residual enzyme activity, which are inversely related to the severity of clinical manifestations.

Distinct phenotypes have been described, based on age at onset, organ involvement, severity, and rate of progression (Table 1). As a general rule, the earlier the onset of symptoms, the faster the rate of progression. Traditionally, PD patients have been classified into distinct categories, including an early onset classical form, early onset intermediate phenotypes, and the attenuated late onset juvenile and adult forms. Although it is now clear that the wide spectrum of clinical manifestations should be considered a phenotypic continuum, the traditional classification of the phenotypes is useful to define the natural history of the disease and to assess the efficacy of therapeutic strategies. GAA activity may range from complete deficiency $(<1 \%)$ in the severe classical forms, to partial (up to 30\%) deficiency in milder late-onset forms. More than 200 different $G A A$ gene mutations have been reported to date. Some of them are recurrent ${ }^{1}$ and show a specific ethnic distribution; others are pan-ethnic. In most cases a correlation between the type of mutation and the residual enzyme activity can be established and, based on these data, genotype/phenotype correlations have been established. ${ }^{1}$ Although in PD patients glycogen storage is ubiquitous, with consequent multi-systemic manifestations, the clinical picture is mainly due to heart and skeletal muscle involvement. In the classic infantile form the heart is characteristically affected, while symptoms of children and adults with a non-classic presentation are predominantly related to the skeletal muscle dysfunction, resulting in both mobility and respiratory problems.

PD has been untreatable until 2006 when enzyme replacement therapy (ERT) with recombinant human GAA (rhGAA) has been
Correspondence: Giancarlo Parenti, Department of Translational Medical Sciences, Federico II University, Via S. Pansini 5, 80131, Naples, Italy. E-mail: parenti@unina.it

Key words: Pompe disease, glycogen storage disease type II, acid $\alpha$-glucosidase, acid maltase, metabolic myopathy.

Conflict of interests: GP has received travel support from Genzyme; GA has received unrestricted research grants, honoraria and travel support from Amicus, Genzyme, Shire, BioMarin and Actelion; SF has received travel support from Genzyme and Actelion.

Received for publication: 9 January 2013

Revision received: 15 February 2013.

Accepted for publication: 18 February 2013.

This work is licensed under a Creative Commons Attribution NonCommercial 3.0 License (CC BYNC 3.0).

(C) Copyright G. Parenti et al., 2013

Licensee PAGEPress, Italy

Cardiogenetics 2013; 3(s1):e5

doi:10.4081/cardiogenetics.2013.s1.e5

approved, in addition to supportive therapies, for treatment of PD. Although the efficacy of ERT has been established in infantile patients, long-term follow-up of patients with various phenotypes reveals that efficacy is limited in some patients and does not completely correct the disease manifestations. Based on improved understanding of disease pathophysiology, new therapeutic strategies are being developed to overcome the ERT limitations.

\section{Classic infantile disease}

\section{The point of view of the pediatrician}

The classic infantile-onset form of PD is the severe end of the clinical spectrum, having an incidence of 1 in 138,000 among Caucasians.

Severe mutations of $G A A$ gene cause complete or near-complete enzyme deficiency, resulting in a clearly defined clinical course, characterized by early onset of clinical manifestations in the first weeks/months of life with typical cardiac involvement, which may begin in utero and be evident since antenatal life. $^{2}$

Disease manifestations are mainly due to the skeletal muscle and heart involvement with hypotonia, generalized muscle weakness and hypertrophic cardiomyopathy. As the most affected skeletal muscles are the respiratory and proximal muscles of the limbs, patients usually show severe respiratory problems, characterized by recurrent respiratory infec- 
tions and respiratory failure, in combination with delay in motor development or inability to achieve major motor milestones, despite normal cognition. Other disease manifestations are caused by glycogen accumulation in tissues different from muscle and include hepatomegaly, enlarged tongue, feeding difficulties, malnutrition and failure to thrive. Biochemical investigations reveal hypertransaminasemia with increased creatine phosphokinase (CPK) levels.

Studies of the natural history in untreated infants show that many clinical manifestations are rapidly progressive, with poor prognosis and early death, which commonly occurs in the first year of life from cardiopulmonary complications. ${ }^{3,4}$

Until 2000 the management of PD patients was exclusively based on support treatment. Then, clinical trials showed the efficacy of the enzyme replacement therapy with recombinant GAA, and in 2006, ERT with the recombinant enzyme derived from human Chinese hamster ovary cell lines received marketing approval.

ERT is based on the concept that patients' cells and tissues can internalize the recombinant lysosomal hydrolases, periodically administered by an intravenous route, through the mannose or mannose-6-phosphate receptor pathways. Then recombinant enzymes may be delivered to lysosomes, where they are activated, replacing the function of the defective hydrolases. The feasibility of ERT for PD was shown in cultured skeletal muscle and in mice, although in vivo studies showed that skeletal muscles were not an easy target. In fact, most of the recombinant enzyme, intravenously administered to mice, was detected in liver and spleen, while only a small fraction ended up in heart and even less in skeletal muscles.

The efficacy of ERT has been evaluated in infantile patients, in a series of studies in which they were randomized to receive treatment with either $20 \mathrm{mg} / \mathrm{kg}$ or $40 \mathrm{mg} / \mathrm{kg}$ of rhGAA, which was intravenously administered every 2 weeks. These studies showed a prominent effect on cardiac hypertrophy and function. Additionally, and most strikingly, there was a substantial effect on survival. ${ }^{3}$ Skeletal muscle pathology and function were also improved, but only in individual patients and $50 \%$ of classic infantile patients eventually become ventilator dependent and are unable to walk. $^{5}$ Despite these limitations long-term observation of ERT-treated infantile patients shows that ERT substantially modifies the prognosis of the disease in this form, significantly increasing survival of infantile patients. ${ }^{5}$ This longer survival has highlighted previously unrecognized aspects of the disease, ${ }^{6}$ so more attention has to be paid to identify and monitor various systemic clinical features and co-morbidities in surviving infants and children. In fact, despite symptoms are mainly caused by glycogen accumulation in skeletal, cardiac and smooth muscles, different manifestations occur in other organs and systems, including the central and peripheral nervous system. Hearing abnormalities, reflecting a cochlear and/or conductive pathology, are increasingly recognized in classic infantile patients, who also show speech disorders with reduced intelligibility and hyper nasal resonance. Hearing loss persists during ERT in surviving infants, requiring careful monitoring for early implementation of hearing aids and speech therapy. ${ }^{7,8}$

Feeding difficulties are common in infantile patients and may result from facial hypotonia, macroglossia, tongue weakness, and/or poor oromotor skills. The assessment of swallowing function in classic infantile patients has shown oropharyngeal dysphagia, with poor cough reflex and possible airway invasion. ${ }^{9}$ So, early and regular swallowing assessment and development of a safe feeding plan are recommended to prevent aspiration and pneumonia

Table 1. Main clinical features in different age-related phenotypes.

\begin{tabular}{|c|c|c|c|c|}
\hline & $\begin{array}{l}\text { Classic infantile } \\
\text { phenotype }\end{array}$ & $\begin{array}{l}\text { Non classic infantile } \\
\text { phenotype }\end{array}$ & Juvenile phenotype & Adult phenotype \\
\hline Age at onset & First months of life & $<2$ years & $\begin{array}{l}>2 \text { years } \\
>18 \text { years }\end{array}$ & $<18$ years \\
\hline Heart involvement & $\begin{array}{c}\text { Hypertrophic cardiomyopathy } \\
\text { Arrhythmias } \\
\text { Cardiac failure }\end{array}$ & $\begin{array}{l}\text { Shortened PR interval on ECG } \\
\text { Huge QRS complexes } \\
\text { Absent or mild cardiomyopathy }\end{array}$ & Generally absent & Generally absent \\
\hline $\begin{array}{l}\text { Skeletal muscle and } \\
\text { locomotor system }\end{array}$ & $\begin{array}{l}\text { Severe and progressive muscle } \\
\text { weakness, severe hypotonia } \\
\text { (floppy infant), delayed motor } \\
\text { milestones, spinal gibbus, joint } \\
\text { contractures }\end{array}$ & $\begin{array}{c}\text { Progressive muscle weakness } \\
\text { and hypotonia, delayed motor } \\
\text { milestones, spinal gibbus, } \\
\text { joint contractures }\end{array}$ & $\begin{array}{l}\text { Progressive muscle weakness } \\
\text { (trunk and proximal muscles } \\
\text { of lower limbs), regression of } \\
\text { motor milestones, scoliosis, } \\
\text { lordosis, scapular winging }\end{array}$ & $\begin{array}{l}\text { Difficult walking or climbing } \\
\text { stairs, gait abnormalities, limb } \\
\text { muscles weakness, loss of motor } \\
\text { skills, low back pain, scoliosis, } \\
\text { lordosis, scapular winging, } \\
\text { myopathic pattern on EMG }\end{array}$ \\
\hline $\begin{array}{l}\text { Respiratory } \\
\text { involvement }\end{array}$ & $\begin{array}{l}\text { Recurrent respiratory infections } \\
\text { Respiratory insufficiency, death } \\
\text { due to cardiorespiratory failure }\end{array}$ & $\begin{array}{l}\text { Recurrent respiratory infections } \\
\text { Respiratory insufficiency }\end{array}$ & $\begin{array}{l}\text { Respiratory problems in the } \\
\text { second decade of life }\end{array}$ & $\begin{array}{c}\text { Respiratory infections } \\
\text { Respiratory insufficiency } \\
\text { Sleep apnea } \\
\text { Dyspnea on exercise }\end{array}$ \\
\hline $\begin{array}{l}\text { Neurologic } \\
\text { involvement }\end{array}$ & Abnormal brain myelination & Abnormal brain myelination & $\begin{array}{l}\text { Intracranial aneurysms or } \\
\text { arteriopathy }\end{array}$ & $\begin{array}{l}\text { Intracranial aneurysms or } \\
\text { arteriopathy }\end{array}$ \\
\hline $\begin{array}{l}\text { Gastrointestinal } \\
\text { involvement }\end{array}$ & $\begin{array}{c}\text { Hepatomegaly } \\
\text { Splenomegaly } \\
\text { Feeding difficulties/dysphagia } \\
\text { Poor growth }\end{array}$ & $\begin{array}{c}\text { Hepatomegaly } \\
\text { Splenomegaly } \\
\text { Feeding difficulties/dysphagia } \\
\text { Poor growth }\end{array}$ & Rare organomegaly & Rare organomegaly \\
\hline Head and neck & $\begin{array}{l}\text { Macroglossia } \\
\text { Hearing loss }\end{array}$ & $\begin{array}{l}\text { Macroglossia } \\
\text { Hearing loss }\end{array}$ & Rare macroglossia & Rare macroglossia \\
\hline Biochemical findings & $\begin{array}{c}\text { Elevated AST/ALT } \\
\text { Elevated serum CK }\end{array}$ & $\begin{array}{l}\text { Elevated AST/ALT } \\
\text { Elevated serum CK }\end{array}$ & $\begin{array}{l}\text { Elevated AST/ALT } \\
\text { Elevated serum CK }\end{array}$ & $\begin{array}{c}\text { Elevated AST/ALT } \\
\text { Elevated serum CK }\end{array}$ \\
\hline Prognosis & Death before 12 months of life & Variable & Variable & Variable \\
\hline
\end{tabular}

ECG, electrocardiogram; EMG, electromyogram; AST, aspartate aminotransferase; ALT, alanine transaminase; CK, creatine kinase. 
that represent high-risk complications for all patients. $^{10}$

\section{The point of view of the cardiologist}

From a cardiologist's point of view, Pompe disease is one of the most important (and devastating) cause of hypertrophic cardiomyopathy in newborns and neonates (infantile classic form). ${ }^{11}$ In 1932, a Dutch pathologist, Dr. Johannes Pompe, described the case of a 7month old infant who died suddenly for a severe idiopathic hypertrophy of the heart. ${ }^{12}$ Dr. Pompe first demonstrated, in the heart and other organs, a vacuolar storage of glycogen (cardiomegalia glycogenica).

Along with muscular hypotonia (floppy baby), macroglossia, and organomegalia, cardiomyopathy and conduction disorders represent typical features (clinical markers) of the disease in its classic infantile presentation.

Cardiomyopathy is generally of the hypertrophic type, with a significant thickening of the interventricular septum (asymmetric hypertrophy), or most frequently of both the septum and the left and right ventricular free walls (concentric hypertrophy). A left outflow tract obstruction (favored by a systolic anterior motion of the anterior mitral leaflet toward the interventricular septum) may be present in a subgroup of patients with severe hypertrophy and small left ventricular cavity. Both diastolic and systolic dysfunction of the left and right heart can be observed. Levine et al. showed a ventricular remodeling with rapid regression of left ventricular hypertrophy and preserved systolic function in response to ERT in most of the patients treated ${ }^{13}$ (Figures 1 and 2).

Not only cardiac myocytes, but also the special cells of the conduction system (particularly, the A-V node and the His-bundle cells) are interested by glycogen storage in Pompe disease. This represents the histological background of classical electrocardiographic (ECG) anomalies commonly observed in Pompe disease, such as ventricular pre-excitation patterns (short PR, delta waves), various degrees of atrio-ventricular blocks, and intraventricular conduction abnormalities (bundle branch blocks). The pathogenesis of ventricular preexcitation in Pompe disease is unknown, though is clear that the pattern does not reflect the presence of an anatomic accessory pathway (as in the classic Wolf-Parkinson-White syndrome, WPW). ${ }^{14}$ To explain this phenomenon, various hypothesis have been raised, including: i) a direct insulating effect of the glycogen on the conduction system; ii) an indirect insulating effect of the glycogen on the conduction system, by the anatomic interruption of the annulus fibrosus (which acts as an electric insulate between the atria and the ventricles). ${ }^{15}$

Various forms of metabolic or mitochondrial cardiomyopathies may overlap the clinical pres-
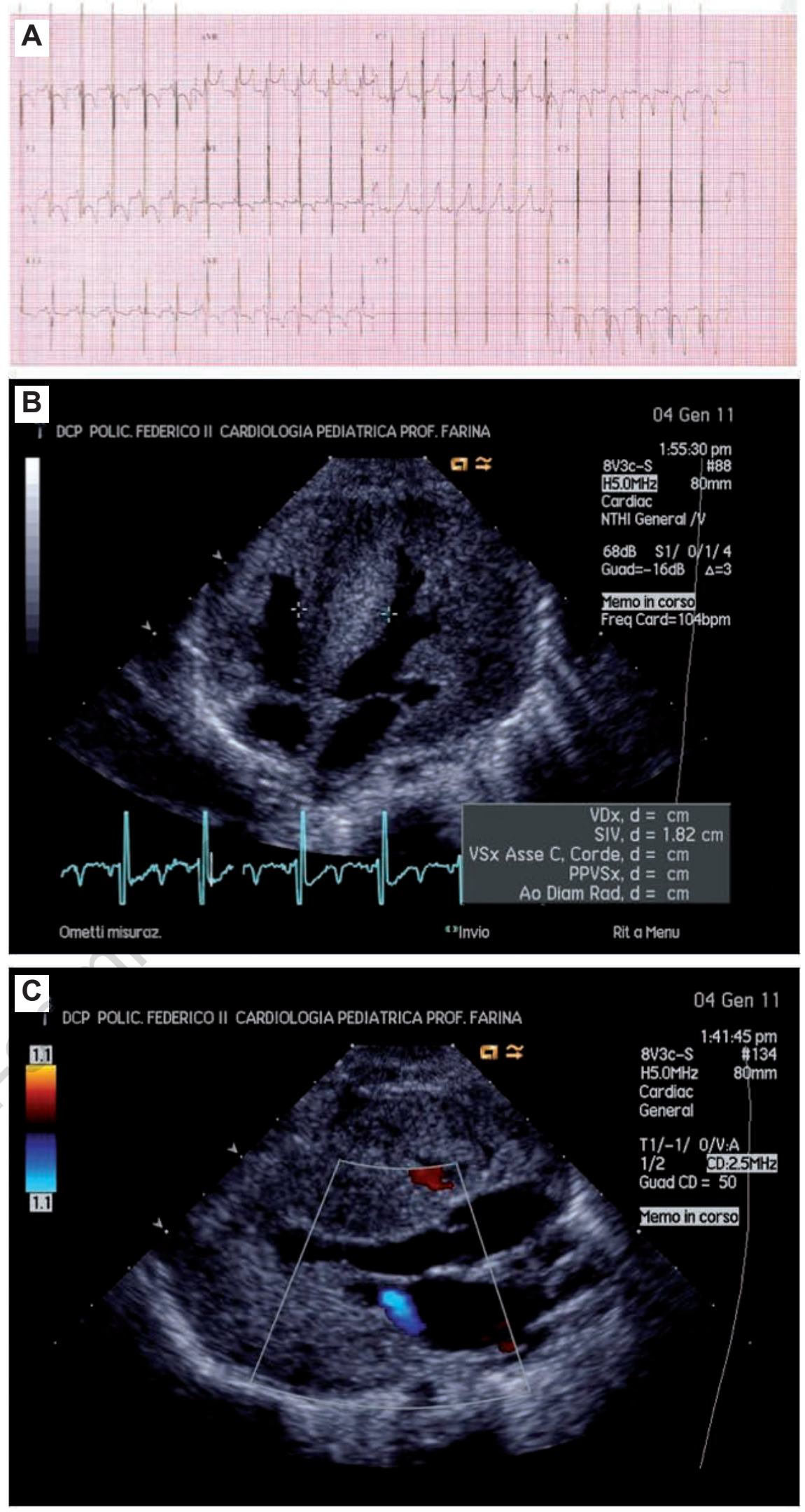

Figure 1. Patient No. 3: Severe, infantile hypertrophic cardiomyopathy (HCM) with systolic dysfunction, on diuretics and ace-inhibitors. Enzyme replacement therapy started late ( 6 months of life). The patient died for heart failure before 1 year of age. A) Electrocardiogram showing short PR, left ventricular hypertrophy and negative $T$ waves in precordial leads. B and C) Severe, biventricular HCM with systolic dysfunction and mitral regurgitation. 
entation of Pompe cardiomyopathy. ${ }^{15}$ Some clinical features may be of help to guide the final diagnosis. A clinical presentation with cardiomyopathy and encephalomyopathy, or cardiomyopathy and metabolic acidosis (with or without hypoglycemia), may suggest a mitochondrial cardiomyopathy. The increase of lactate and lactate/pyruvate ratio (normal: <15:1; abnormal: 25:1) is a hallmark of the disease. A presentation with cardiomyopathy and hypoglycemia is a typical presentation of a spectrum of metabolic disorders, ranging from benign form (i.e. infant of diabetic mothers), to complex metabolic diseases (i.e. beta-oxidation deficit), requiring the evaluation of a series of biochemical parameters (plasma ketones, insulin, free fatty acids, carnitine, etc.).

\section{Non-classic childhood disease}

\section{The point of view of the pediatrician}

Intermediate phenotypes of PD have also been described and characterized. Even within the infantile category, there exists a wide range of severity and some patients with the so called non-classic or muscular variant infantile-onset phenotype, may display symptoms before 1 year of age, with clinical manifestations which are similar to the classic infantile patients but without acute cardiomyopathy and with slower progression.

Differently from the infantile onset, which is characterized by marked organ involvement, the childhood-onset form usually presents later than the infantile, with muscle weakness and elevation of CPK levels, mimicking those of progressive muscular dystrophy, in absence of severe cardiomyopathy.

While the benefits of ERT for the infantile form have been well documented, particularly on survival and heart involvement, its efficacy in childhood-onset patients is widely variable. ${ }^{16,17}$ The results of clinical trials in lateonset patients supported the efficacy of ERT in improving motor functions (mainly assessed as walking distance) and in stabilizing pulmonary function, but improvement was limited in some patients. ${ }^{18,19}$ Skeletal muscle (which is the main tissue involved in the late-onset forms) responds poorly to treatment in comparison with the heart. Effects are thought to depend on the extent of muscle damage at start of treatment and the best response has been obtained in those patients who were in the best conditions. Survival, in patients who had been diagnosed by newborn screening, was significantly improved, compared with those treated later. ${ }^{20}$

Hence, considering that ERT is a safe and effective treatment, early identification of affected children becomes crucial to ideally start treatment before extensive tissue damage develops.
A simple diagnostic algorithm has been proposed by national and international guidelines in infantile forms, ${ }^{21,22}$ firstly performing GAA enzymatic assay in patients showing hypertrophic cardiomyopathy, in combination with generalized hypotonia, hypertransaminasemia and increased CPK. Diagnosis should be confirmed by molecular analysis of $G A A$ gene since identification of causative mutations is also helpful for familial screening and prenatal diagnosis in future pregnancies.

Otherwise, differential diagnosis in lateonset forms may be difficult and may determine delay in the diagnosis of PD. Recently innovative diagnostic methods, such as meas- urement of GAA activity in dried blood spots by tandem mass spectroscopy, can be used to investigate suspected patients and in newborns screening programs. ${ }^{23,24}$

A tetra glucose oligomer designated as Glc4 has been shown to be elevated in both urine and plasma of PD patients and it might be used as a non-invasive marker for diagnosis and monitoring of therapeutic response, ${ }^{25}$ although a consistent interpretation of the data has not been reached yet.

As the muscle weakness has a strong impact on multisystemic functions, the management of children affected by PD requires a multidisciplinary team, coordinated by a metabolic

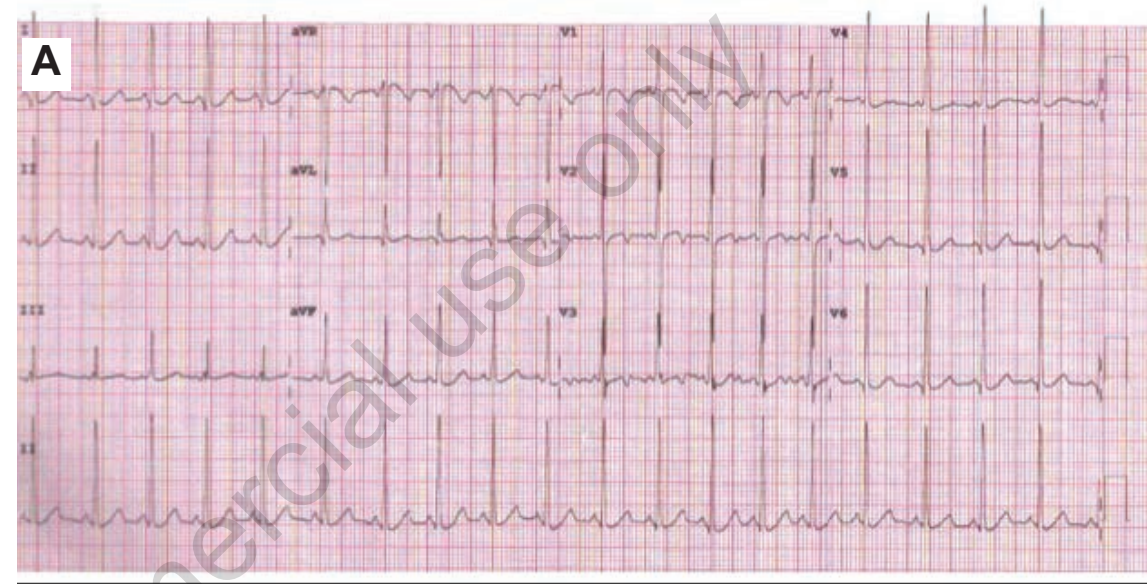

\section{B}

DCP POLIC. FEDERICO II CARDIOLOGIA PEDIATRICA PROF, FARINA
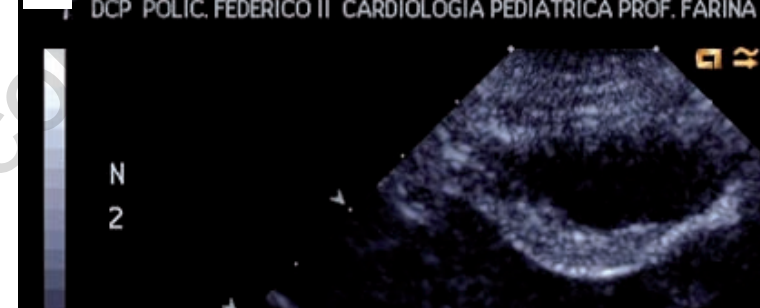

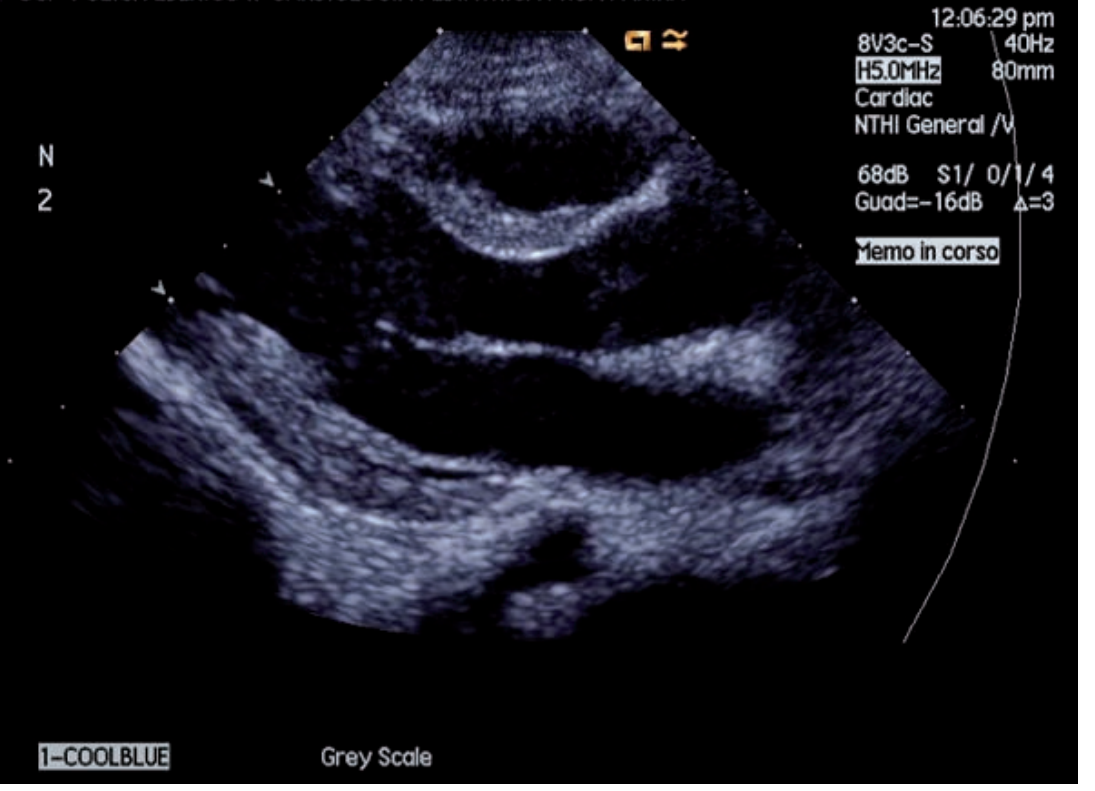

Figure 2. Patient No. 1: Severe, infantile hypertrophic cardiomyopathy with systolic dysfunction, on diuretics, ACE-inhibitors, and digitalis. Enzyme replacement therapy started at 3 months of life. A progressive cardiac ventricular remodeling has been observed during follow-up. A) Electrocardiogram showing short PR, mild signs of left ventricular hypertrophy without repolarization abnormalities. B) Normalization of wall thickness, ventricular diameters, and left ventricular systolic function. 
pediatrician or a pediatric neurologist, for early identification and supportive treatment of multi-organ complication. ${ }^{21,26}$

Respiratory management is crucial in PD patients, as most of them experience some forms of respiratory involvement, due to the occurrence of several factors as muscle weakness, thoracic deformities with scoliosis and reduced thoracic compliance, poor cough and recurrent infections. These features may concur to the development of respiratory failure (which is the most common cause of premature death) and some patients need ventilatory support. Other support interventions may include special forms of physical therapy to strengthen weakened respiratory muscles, as well as aggressive management of infections.

Physical therapy has an important role to maximize physical function and to prevent contractures and deformity, particularly in those children who are now surviving longer with ERT. ${ }^{27}$

Due to the common finding of growth failure and feeding difficulties in children with PD, nutritional intervention is required to ensure that all macro and micronutrient needs are met. A nutritional approach based on dietary supplementation of proteins and/or L-alanine has also been proposed ${ }^{28}$ in addition to ERT.

Finally, based on the new emerging phenotype of long-term survivals, appropriate followup should be established for identifying and monitoring different clinical aspects as hearing loss, arrhythmias, and dysphagia with risk for aspiration and osteopenia. ${ }^{29}$

\section{The point of view of the cardiologist}

In the non-classic form of Pompe disease, the clinical picture is generally less severe in all its different systemic aspect, including muscle weakness, hypertrophic cardiomyopathy and conduction disorders, and in some cases macroglossia and organomegalia.

Pediatric cardiologists recognize conduction abnormalities and ECG signs of ventricular hypertrophy as part of the disease spectrum. Hypertrophic cardiomyopathy is generally less severe and progressive, lacking the left ventricular obstruction and the systolic dysfunction that significantly worsen the classic phenotype in its classic infantile onset. Nevertheless, the clinical presentation may be extremely various, and the heart may represent an isolated target of the disease expression, in selected cases. Indeed, Suzuki et al ${ }^{30}$ reported on a male who developed cardiomyopathy at 12 years of age and died of heart failure at age 15 years without any clinical and/or histological sign of skeletal myopathy.

The non-classic infantile form of Pompe disease should be distinguished from overlapping syndromes, mitochondrial or metabolic cardiomyopathies. ${ }^{31}$ Ventricular pre-excitation on the ECG and the presence of left ventricular hypertrophy in children are common features of other storage diseases (AMP kinase disease, Danon disease), and mitochondrial disorders (MELAS, MERFF). Particularly, Danon disease is an X-linked glycogen storage disorder due to the absence of the LAMP-2, lysosome-associated membrane protein 2 (evidenced by immunohistochemistry or by genetic sequencing). Cognitive impairment and retinitis pigmentosa, when present, may be distinctive feature in patients with Danon disease.

\section{Adult-onset disease}

\section{The point of view of the neurologist}

The adult form of Pompe disease is not an autonomous entity with respect to those classic and juvenile, but differs from these for the higher acid- $\alpha$-glucosidase (AAG) residual activity in body tissues and a lower rate of lysosomal glycogen accumulation leading to a later onset of clinical manifestations. Having an incidence of 1/57,000/year, it presents after age 18 , by convention, but it is first diagnosed during the third or the fourth decades of life. ${ }^{32,33}$ Historically, disease onset can be dated 3-10 years back, almost always. This time interval form first symptoms to diagnosis is mainly related to the rather unspecific and slow progressive symptoms which startle neither the patient nor the doctor. Furthermore, many practitioners ignore the existence of the adult form of the disease.

The disease manifests as a proximal myopathy with variable severity from patient to patient. Most patients report reduced endurance during physical activity, lumbar pain, muscle pain and cramps at the lower limbs since their childhood. ${ }^{34}$ Tiredness even after moderate exercise and frequent downfall are reported. Running and walking, climbing up and down stairs, getting up from easy chairs, and rising up from a lying position are progressively impaired due to changes in pelvic girdle and proximal lower limbs muscles. Shoulder girdle and upper limbs are involved later, usually to a lesser extent. Paraspinal muscles weakness, lumbar hyperlordosis, scoliosis and waddling gait are common. Thirteen percent of the patients early complain with nocturnal respiratory difficulties, snoring, and headache at awakening and recurrent pulmonary infections due to the involvement of diaphragm and intercostals muscles, typical of Pompe disease. Patients should always be asked if they suffer from orthopnea, exertional dyspnea and ineffective coughing. An easy test to assess reduced vital capacity is counting out loud during the expiratory phase, first supine and then sitting. A lower supine count should prompt physicians to refer the patient to the pulmonologist. Creatine kinase (CK) is always mildly increased in serum. Differential diagnosis may be challenging with the limb-girdle dystrophies 2A and 2I, and with Danon disease. Diagnostic algorithms for the adult form of Pompe disease $^{35}$ indicate clinical evaluation, serum CK measurement, electromyography/ electroneurography, spirometry, and, when indicated, a muscle biopsy which will show a vacuolar myopathy with accumulation of glycogen. AAGactivity less than $30 \%$ of normal in skeletal muscle, fibroblasts, or lymphocytes further address the diagnosis which is finally confirmed by mutational analysis of the $G A A$ gene.

\section{The point of view of the pulmonologist}

Respiratory failure is the major cause of morbidity and mortality among patient affected with the juvenile or adult form of Pompe disease. For $30 \%$ of adult patients with glycogenosis type II, respiratory insufficiency may be the presenting clinical feature. ${ }^{23}$

The development of respiratory failure in patients with Pompe disease is often insidious, particularly when the inability to walk progresses in parallel with loss of ventilatory capacity. Assessment is based on the pattern of nonspecific symptoms, such as fatigue, lethargy, or difficult in concentration. Dyspnea and orthopnea are often late findings in patients with usually severe functional impairment due to peripheral muscle weakness, although orthopnea may be associated with diaphragmatic paralysis. As the disease progresses, tachypnea at rest may become a typical manifestation. ${ }^{4}$

Pulmonary function tests, including spirometry and lung volumes, are routinely performed during the evaluation of patients with neuromuscular disease. A specific evaluation of respiratory muscle strength is mandatory as these tests are both sensitive and highly prognostic. A high-negative maximal inspiratory pressure (MIP) result $(>80)$ or a high-positive maximal expiratory pressure (MEP) result $(>90 \mathrm{~cm}$ $\mathrm{H}_{2} \mathrm{O}$ ) excludes clinically relevant inspiratory or expiratory muscle weakness. Possible discrepancies (particularly in bulbar patients) between MIP and sniff nasal inspiratory pressure justify performing both measurements and selecting the highest pressure. ${ }^{36,37}$

Because of the inadequacy of inspiratory muscle function, a well-known pattern of restrictive ventilatory defect can be detected by pulmonary function tests. When the vital capacity falls below $55 \%$ of predicted normal values, the onset of insidiously progressive hypercapnia is likely. ${ }^{38} \mathrm{~A}$ significant difference between upright and recumbent lung volumes and flow rates has been reported frequently. In particular, a measurement of the closing volume (CV) in a sitting position proved abnormal ( $<80 \%$ predicted), requires the execution of the examination in the supine position to assess the possible 
weakness of the diaphragm. A fall of CV $>20 \%$, going from sitting to supine position, is a sign of severe weakness of the diaphragm.

Bulbar, inspiratory, and expiratory muscles are needed for effective coughing. The reduced efficiency of cough exposes the patient to respiratory infections. Inadequate expiratory muscle function may cause bronchial mucous encumbrance, frequently complicated by pneumonia, atelectasis and acute respiratory failure.

Cough peak expiratory flow (CPEF) is the single most important factor in determining whether the ability to eliminate bronchial secretion is well preserved. A value $\mathrm{CPEF}<270$ $\mathrm{L} / \mathrm{min}$ or $\mathrm{MEP}<60 \mathrm{~cm} \mathrm{H}_{2} \mathrm{O}$, indicating compromised cough efficiency.

Arterial blood gas analysis is important to be aware that overt ventilatory failure can occur abruptly, although this measurement of arterial blood gas composition is not a reliable indicator of this danger.

Patient with glycogenosis type II are susceptible to the development of sleep-disorderedbreathing, with the commonest form being hypoventilation. Hypoventilation is caused by a reduction in the tidal volume, particularly during REM sleep. The nocturnal hypoventilation usually precedes the onset of a stable alveolar hypoventilation and daytime hypercapnia. Obstructive events during sleep have been reported in patients with neuromuscular disease due to pharyngeal or laryngeal muscle weakness, which predisposes to upper airway collapse.

Nocturnal oxymetry is first level examination and, when it is not diagnostic, polysomnography should be performed. Overnight polysomnography is advisable for patients who develop symptoms and signs of sleep-wake abnormality or nocturnal respiratory failure.

Patients with ventilatory muscle failure require ventilatory support. Nocturnal nasal continuos positive airway pressure can be considered only in patients with neuromuscular obstructive sleep apnea, but without clinical and instrumental evidence of alveolar hypoventilation. Long-term non-invasive positive pressure ventilation is required when spontaneous respiratory muscle efforts are unable to sustain adequate alveolar ventilation, causing chronic-stable, or slowly progressive ventilatory failure.

In case of severely impaired swallowing, leading to chronic aspiration and repeated pneumonia and the need for around-the-clock $(>20 \mathrm{~h}$ ) ventilatory support tracheostomy and invasive mechanical ventilation are required. ${ }^{39}$

\section{The point of view of the cardiologist}

Cardiac involvement is a rare finding in adult-onset disease. ${ }^{31}$ On the other hand, the cardiac phenotype of adults with acid maltase deficiency is still poorly characterized.

Reports of heart abnormalities in adults with
Pompe disease are sparse. Recently, a relatively large cohort of adults ( 87 patients, median age 44 years old, 51\% males) with Pompe disease has been described. ${ }^{40} \mathrm{~A}$ short PR interval was present in $10 \%$ of the patients, $7 \%$ showed a decreased left ventricular systolic function, and $5 \%$ had cardiomyopathy (increased left ventricular mass by echocardiography). Interestingly, no changes in cardiovascular status associated with ERT were observed.

In adults with Pompe disease, severe vacuolization of vascular smooth muscle with accumulation of glycogen, mainly involving large and small cerebral arteries with aneurysm formation, have been reported. ${ }^{41,42}$ Güngör et al. ${ }^{43}$ described survival of 268 adult patients with Pompe disease, prospectively observed between 2002 and 2009. Among 34 deaths, the underlying cause was recognized in 9 patients, including 1 patient who died for aortic dissection. Smooth muscle involvement has been confirmed by the finding of an increased aortic stiffness in adults with Pompe disease,${ }^{44}$ which is related to glycogen storage in the vessel wall, causing reduced vascular elasticity. These findings deserve future investigations.

\section{Conclusions and future perspectives}

PD is a complex metabolic disease with a heterogeneous clinical spectrum and severity, related to the varying levels of residual enzyme activity. Overall, the studies on ERT in PD support the efficacy of this approach in improving survival and function or in stabilizing the disease course.

However, limitations of ERT are becoming evident and the beneficial effect may be unpredictable, mainly in late-onset patients. This could be due to the relative refractoriness of skeletal muscles to therapy, based on the occurrence of several factors such as the preferential uptake of the recombinant enzyme by liver, ${ }^{45}$ the resistance of type IIb muscle fibers to therapy and the large mass of skeletal muscle, in combination with the relative deficiency of the mannose-6-phosphate receptors in muscle cells ${ }^{46}$ and its intracellular mislocalization, which results in less efficient internalization and lysosomal trafficking of rhGAA. Furthermore, the variability of the response to the ERT among patients may be related to their immunological status, with formation of neutralizing antibodies to the recombinant GAA in patients bearing mutations that cause the absence of a protein product (cross reactive immunological material-negative). ${ }^{47}$

The disease stage and the duration, together with the age at start of treatment, are also important factors.

Considering the overall limitations of ERT, together with its high costs, innovative therapeutic approaches are now under development.

The different therapeutic strategies are mainly based on new rhGAA preparations (chemically modified to improve muscle targeting); enzyme enhancement with small molecule pharmacological chaperones; the combination of chaperones and ERT; the substrate reduction and the gene therapy. Among the different strategies, enzyme enhancement with small molecule (pharmacological chaperones) apparently represents a very attractive and feasible approach, based on the concept that some gene mutations may cause protein misfolding and degradation of the mutated enzyme, and that small molecules chaperones may assist in the correct folding of the mutated GAA, improving its stability and lysosomal trafficking.

Two imino sugars, 1-deoxynojirimycin (DNJ) and its alkylated derivative $\mathrm{N}$ butyldeoxynojirimycin (NB-DNJ) are proved to be effective in enhancing GAA residual activity in fibroblasts from PD patients carrying specific mutations of the $G A A$ gene. ${ }^{48-50}$

Although the marketing approval of NB-DNJ for treatment of Gaucher and Niemann-Pick type $\mathrm{C}$ diseases represents an advantage for its clinical use in PD, the feasibility of this approach appears limited, considering that only a small percentage of PD patients (about $10-15 \%$ ) with responsive mutations could benefit from this therapy.

The results of recent studies showed the effects of the chaperone molecule NB-DNJ on ERT ${ }^{51}$ possibly correcting inappropriate trafficking and/or decreased stability of the wild type recombinant enzymes. Based on these observations a different approach has been proposed, using the combination of chaperones and ERT, which may have a synergistic effect, considered to be particularly useful in reaching therapeutic levels of recombinant enzyme in tissues poorly responding to therapy. To evaluate the efficacy of this approach, based on the combination of ERT with NB-DNJ in PD patients, a multicenter clinical trial is now in progress in Italy (Miglu3, EudraCT Number: 2010-024647-32, supported by Fondazione Telethon).

A better understanding about the pathophysiology of the disorder might highlight new therapeutic targets for additional strategies, aimed at the correction of secondary cellular abnormalities (for example the modulation of autophagy or the reduction of protein degradation by the so-called proteostasis regulators) and possibly the combination with different approaches.

Finally, the results of preclinical studies, exploring the feasibility of gene therapy in PD, seem to be promising, although several issues remain to be addressed before gene therapy can be translated to clinics. 


\section{References}

1. Hermans MM, van Leenen D, Kroos MA, et al. Twenty-two novel mutations in the lysosomal $\alpha$-glucosidase gene (GAA) underscore the genotype-phenotype correlation in glycogen storage disease type II. Hum Mutat 2004;23:47-56.

2. Hamdan MA, El-Zoabi BA, Begam MA, et al. Antenatal diagnosis of pompe disease by fetal echocardiography: impact on outcome after early initiation of enzyme replacement therapy. J Inherit Metab Dis 2010. [Epub Ahead of Print].

3. Van den Hout HM, Hop W, van Diggelen OP, et al. The natural course of infantile Pompe's disease: 20 original cases compared with 133 cases from the literature. Pediatrics 2003;112:332-40.

4. Kishnani PS, Hwu WL, Mandel H, et al. A retrospective, multinational, multicenter study on the natural history of infantileonset Pompe disease. J Pediatr 2006;148: 671-6.

5. Kishnani PS, Corzo D, Leslie ND, et al. Early treatment with alglucosidase $\alpha$ prolongs long-term survival of infants with Pompe disease. Pediatr Res 2009;66:329-35.

6. Prater SN, Banugaria SG, Dearmey SM, et al. The emerging phenotype of long-term survivors with infantile Pompe disease. Genet Med 2012;14:800-10.

7. Kamphoven JH, de Ruiter MM, Winkel LP, et al. Hearing loss in infantile Pompe's disease and determination of underlying pathology in the knockout mouse. Neurobiol Dis 2004;16:14-20.

8. van Capelle CI, Goedegebure A, Homans NC, et al. Hearing loss in Pompe disease revisited: results from a study of 24 children. J Inherit Metab Dis 2010;33: 597-602.

9. Jones HN, Muller CW, Lin M, et al. Oropharyngeal dysphagia in infants and children with infantile Pompe disease. Dysphagia 2010;25:277-83.

10. van Gelder CM, van Capelle CI, Ebbink BJ, et al. Facial-muscle weakness, speech disorders and dysphagia are common in patients with classic infantile Pompe disease treated with enzyme therapy. J Inherit Metab Dis 2012;35:505-11.

11. Elliott P, Andersson B, Arbustini E, et al. Classification of the cardiomyopathies: a position statement from the European Society Of Cardiology Working Group on Myocardial and Pericardial Diseases. Eur Heart J 2008;29:270-6.

12. Pompe JC. Over idiopathische hypertrophie van het hart. Ned. Tijdschr. Geneeskd 1932;76:304-12.

13. Levine JC, Kishnani PS, Chen YT, et al. Cardiac remodeling after enzyme replace- ment therapy with acid $\alpha$-glucosidase for infants with Pompe disease. Pediatr Cardiol 2008;29:1033-42.

14. Arad M, Moskowitz IP, Patel VV, et al. Transgenic mice overexpressing mutant PRKAG2 define the cause of WolffParkinson-White syndrome in glycogen storage cardiomyopathy. Circulation 2003; 107:2850-6.

15. Limongelli G, Elliott PM. The genetics of heart failure. Oxford Texbook of Heart Failure 0xford: Oxford University Press; 2011.

16. Case LE, Koeberl DD, Young SP, et al. Improvement with ongoing enzyme replacement therapy in advanced lateonset Pompe disease: a case study. Mol Genet Metab 2008;95:233-5.

17. Merk T, Wibmer T, Schumann C, Krüger S. Glycogen storage disease type II (Pompe disease)-influence of enzyme replacement therapy in adults. Eur J Neurol 2009;16: 274-7.

18. Strothotte S, Strigl-Pill N, Grunert B, et al. Enzyme replacement therapy with alglucosidase alfa in 44 patients with late-onset glycogen storage disease type 2: 12-month results of an observational clinical trial. J Neurol 2010;257:91-7.

19. van der Ploeg AT, Clemens PR, Corzo D, et al. A randomized study of alglucosidase alfa in late-onset Pompe's disease. $\mathrm{N}$ Engl J Med 2010;362:1396-406.

20. Chiang SC, Hwu WL, Lee NC, et al. Algorithm for Pompe disease newborn screening: results from the Taiwan screening program. Mol Genet Metab 2012;106: 281-6.

21. Bembi B, Cerini E, Danesino C, et al. Management and treatment of glycogenosis type II. Neurology 2008;71 Suppl 2:S12-36.

22. Bembi B, Cerini E, Danesino C, et al. Diagnosis of glycogenosis type II. Neurology 2008;71 Suppl 2:S4-11.

23. Dajnoki A, Mühl A, Fekete G, et al. Newborn screening for Pompe disease by measuring acid $\alpha$-glucosidase activity using tandem mass spectrometry. Clin Chem 2008;54:1624-9.

24. la Marca G, Casetta B, Malvagia S, et al. New strategy for the screening of lysosomal storage disorders: the use of the online trapping-and-cleanup liquid chromatography/mass spectrometry. Anal Chem 2009;81:6113-21.

25. Manwaring V, Prunty H, Bainbridge K, et al. Urine analysis of glucose tetrasaccharide by HPLC; a useful marker for the investigation of patients with Pompe and other glycogen storage diseases. J Inherit Metab Dis 2012;35:311-6.

26. Kishnani PS, Steiner RD, Bali D, et al. Pompe disease diagnosis and management guideline. Genet Med 2006;8:267-88.
27. Case LE, Beckemeyer AA, Kishnani PS. Infantile Pompe disease on ERT: update on clinical presentation, musculoskeletal management, and exercise considerations. Am J Med Genet C Semin Med Genet 2012;60:69-79.

28. Bodamer OA, Leonard JV, Halliday D. Dietary treatment in late-onset acid maltase deficiency. Eur J Pediatr 1997;156 Suppl 1:S39-42.

29. van den Berg LE, Zandbergen AA, van Capelle CI, et al. Low bone mass in Pompe disease: muscular strength as a predictor of bone mineral density. Bone 2010;47: 643-9.

30. Suzuki Y, Tsuji A, Omura K, et al. Km mutant of acid $\alpha$-glucosidase in a case of cardiomyopathy without signs of skeletal muscle involvement. Clin Genet 1988;33: 376-85.

31. Limongelli G, Fratta F. Cardiovascular involvement in Pompe disease. Acta Myol 2011;30:202-3.

32. van der Beek NA, Hagemans ML, van der Ploeg AT, et al. Pompe disease (glycogen storage disease type II): clinical features and enzyme replacement therapy. Acta Neurol Belg 2006;106:82-6.

33. Müller-Felber W, Horvath R, Gempel K, et al. Late onset Pompe disease: clinical and neurophysiological spectrum of 38 patients including long-term follow-up in 18 patients. Neuromuscul Disord 2007;17: 698-706.

34. Van der Ploeg AT, Reuser AJJ. Lysosomal storage disease 2. Pompe disease. Lancet 2008;372:1342-53.

35. American Association of Neuromuscular \& Electrodiagnostic Medicine. Diagnostic criteria for late-onset (childhood and adult) Pompe disease. Muscle Nerve 2009; 40:149-60.

36. van der Ploeg AT. Monitoring of pulmonary function in Pompe disease: a muscle disease with new therapeutic perspectives. Eur Respir J 2005;26:984-5.

37. Hagemans ML, Winkel LP, van Doorn PA, et al. Clinical manifestation and natural course of late onset Pompe's disease in 54 Dutch patients. Brain 2005;128:671-7.

38. Hagemans ML, Hop WJ, van Doorn PA, et al. Course of disability and respiratory function in untreated late onset Pompe disease. Neurology 2006;66:581-3.

39. Caputi M, De Michele F, Maddalena A, et al. Linee guida Regione Campania ventilazione meccanica domiciliare adulti e pediatrica. Regione Campania; 2008. Available from: http://insufficienzarespiratoria.aiponet.it [In Italian].

40. Forsha D, Li JS, Smith PB, et al. Cardiovascular abnormalities in late onset Pompe disease and response to enzyme replacement therapy. Genet Med 2011; 13:625-31. 
41. Makos MM, McComb RD, Hart MN, Bennett DR. $\alpha$-glucosidase deficiency and basilar artery aneurysm: report of a sibship. Ann Neurol 1987;22:629-33.

42. Kretzschmar HA, Wagner H, Hübner G, et al. Aneurysms and vacuolar degeneration of cerebral arteries in late-onset acid maltase deficiency. J Neurol Sci 1990;98: 169-83.

43. Güngör D, de Vries JM, Hop WC, et al. Survival and associated factors in 268 adults with Pompe disease prior to treatment with enzyme replacement therapy. Orphanet J Rare Dis 2011;6:34.

44. Nemes A, Soliman OII, Geleijnse ML, et al. Increased aortic stiffness in glycogenosis type 2 (Pompe's disease). Int J Card 2007;120:138-41.
45. Raben N, Danon M, Gilbert AL, et al. Enzyme replacement therapy in the mouse model of Pompe disease. Mol Genet Metab 2003;80:159-69.

46. Wenk J, Hille A, von Figura K. Quantitation of Mr 46000 and Mr 300000 mannose 6phosphate receptors in human cells and tissues. Biochem Int 1991;23:723-31.

47. Douillard-Guilloux G, Richard E, Batista L, Caillaud C. Partial phenotypic correction and immune tolerance induction to enzyme replacement therapy after hematopoietic stem cell gene transfer of $\alpha$-glucosidase in Pompe disease. $\mathrm{J}$ Gene Med 2009;11:279-87.

48. Parenti G, Zuppaldi A, Gabriela Pittis M, et al. Pharmacological enhancement of mutated $\alpha$-glucosidase activity in fibrob- lasts from patients with Pompe disease. Mol Ther 2007;15:508-14.

49. Okumiya T, Kroos MA, Vliet LV, et al. Chemical chaperones improve transport and enhance stability of mutant $\alpha$-glucosidases in glycogen storage disease type II. Mol Genet Metab 2007;90:49-57.

50. Flanagan JJ, Rossi B, Tang K, et al. The pharmacological chaperone 1-deoxynojirimycin increases the activity and lysosomal trafficking of multiple mutant forms of acid $\alpha$-glucosidase. Hum Mutat 2009;30: 1683-92.

51. Porto C, Cardone M, Fontana F, et al. The pharmacological chaperone N-butyldeoxynojirimycin enhances enzyme replacement therapy in Pompe disease fibroblasts. Mol Ther 2009;17:964-71. 